\title{
THE SPECTRUM OF THE LAPLACE OPERATOR FOR A GENERALIZED DOLD MANIFOLD
}

\author{
GR. TSAGAS AND G. DIMOU
}

\section{Introduction:}

Let $(M, g),(N, h)$ be two compact and orientable Riemannian manifolds. Let $\Gamma$ be a finite subgroup of the group of isometries $I(M \times N)$ which acts freely on the manifold $M \times N$. One of the problems of the spectrum is to determine the $\operatorname{Sp}(M \times N / \Gamma)$.

The aim of the present paper is to determine the $S p\left(S^{n} \times \mathbb{P}^{m}(\mathbf{C}) / \mathbb{Z}_{2}\right)$, where $S^{n} \times \mathbb{P}^{m}(\mathrm{C}) / \mathbb{Z}_{2}$ is called Dold manifold.

The whole paper contains four paragraphs.

The second paragraph deals firstly with the spectrum of the manifold ( $M \times$ $N, g \times h)$. It also gives the general theory about $S p(M \times N / \Gamma)$, where $\Gamma$ is a subgroup of $I(M \times N)$ which is isomorphic onto $\mathbb{Z}_{2}$.

The spectrum of the Dold manifold $S^{n} \times \mathbb{P}^{m}(\mathbf{C}) / \mathbb{Z}_{2}$ is computed in the third paragraph.

The last paragraph deals with the conditions such that $\operatorname{Sp}\left(S^{n} \times \mathbb{P}^{m}(\mathbf{C}) / \mathbb{Z}_{2}\right)$ determines the geometry on $S^{n} \times \mathbb{P}^{m}(\mathbf{C}) / \mathbb{Z}_{2}$.

$\S 2$. We consider two compact orientable Riemannian manifolds $(M, g)$ and $(N, h)$. From these manifolds we obtain the manifold $(M \times N, g \times h)$. We have the following diagram:

Received May 21, 1989. 


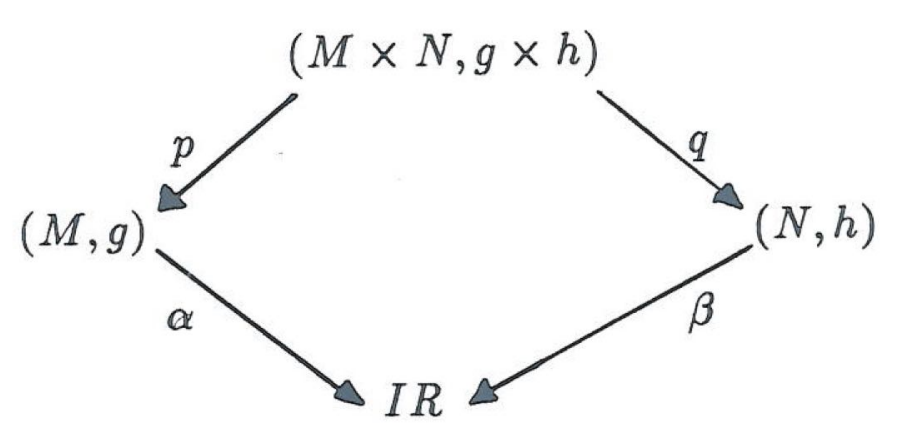

If $\alpha$ and $\beta$ are two functions on $M$ and $N$ respectively, then $\alpha$ op and $\beta$ oq are functions on $M \times N$. We denote by $\triangle^{M \times N}, \triangle^{M}$ and $\Delta^{N}$ the Laplace operators on the Riemannian manifolds $(M \times N, g \times h),(M, g)$ and $(N, h)$ respectively. It can easily be proved

$$
\triangle^{M \times N}[(\alpha o p) \times(\beta o q)]=(\beta o q) \times\left[\triangle^{M}(\alpha) o p\right]+(\alpha o p) \times\left[\triangle^{N}(\beta) o q\right]
$$

If $\alpha$ is an eigenfuction of $\Delta^{M}$ with eigenvalue $\lambda$ and $\beta$ is an eigenfuction of $\Delta^{N}$ with eigenvalue $\mu$, then the relation (2.1) takes the form

$$
\triangle^{M \times N}[(\alpha o p) \times(\beta o q)]=(\lambda+\mu)[(\alpha o p) \times(\beta o q)]
$$

that means $(\alpha o p) \times(\beta o q)$ is an eigenfunction for the Laplace operator $\triangle^{M \times M}$ with eigenvalue $\lambda+\mu$.

Let $\left(M^{\prime}, g^{\prime}\right)$ be a compact and orientable Riemannian manifold with metric $g^{\prime}$. We denote by $C^{\infty}\left(M^{\prime}\right)$ the C-algebra of all differentiable functions on $M^{\prime}$. From $M^{\prime}$ we obtain the $S p\left(M^{\prime}, g^{\prime}\right)=\left\{\lambda / \Delta^{M^{\prime}} f=\lambda f, f \in C^{\infty}\left(M^{\prime}\right), \lambda \in \mathbb{R}\right\}$. We consider the subspace $Q\left(M^{\prime}, g^{\prime}\right)$ of $C^{\infty}\left(M^{\prime}\right)$ defined by

$$
Q\left(M^{\prime}, g^{\prime}\right)=\sum_{\lambda \in S_{P}\left(M^{\prime}, g\right)} Q_{\lambda}\left(M^{\prime}, g^{\prime}\right)
$$

where $Q_{\lambda}\left(M^{\prime}, g^{\prime}\right)$ is the vector subspace of $C^{\infty}\left(M^{\prime}\right)$ consisting of the eigefunctions with eigenvalue $\lambda$.

$Q_{\lambda}\left(M^{\prime}, g^{\prime}\right)$ is called eigen-subspace of $\left(M^{\prime}, g^{\prime}\right)$ and has finite dimension. The subset $Q\left(M^{\prime}, g^{\prime}\right)$ is dense in $C^{\infty}\left(M^{\prime}\right)$ provided with the topology defined by the inner product $\left\langle f_{1}, f_{2}\right\rangle=\int_{M^{\prime}} f_{1} f_{2} d M^{\prime}$, where $d M^{\prime}$ is the volume element on $M^{\prime}$. 
We consider the subspace of $C^{\infty}(M \times N)$ generated by all products of the form $(\alpha o p) \times(\beta o q)$, where $\alpha \in Q(M, g)$ and $\beta \in Q(N, h)$. This vector subspace of $C^{\infty}(M \times N)$ is denoted by $p^{\star} Q(M, g) \otimes g^{\star} Q(N, h)$. This is isomorphic onto the vector space $Q(M, g) \otimes Q(N, h)$. The following relations are true.

$$
p^{\star} Q(M, g) \otimes q^{\star} Q(N, h)=Q(M \times N, g \times h)
$$

$$
\begin{aligned}
& S p(M \times N, g \times h)=\{\lambda+\mu / \lambda \in S p(M, g), \mu \in S p(N, h)\} \\
& Q_{\nu}(M \times N, g \times h)=\sum_{\substack{\lambda \in S p(M, g) \\
\mu \in S p(N, h) \\
\lambda+\mu=\nu}} p^{\star} Q_{\lambda}(M, g) \otimes q^{\star} Q_{\mu}(N, h)
\end{aligned}
$$

Theorem 2.1. Let $(M, g),(n, h)$ be two compact and orientable Riemannian manifolds. Let $\Gamma \simeq \mathbb{Z}_{2}$ be the group of isometries on the manifold $(M \times N, g \times h)$ which acts freely on $M \times N$ such that preserves the seperate coordinate system on the product manifold $M \times N$. Then the eigenfunctions on the manifold $(M \times N / \Gamma, g \times h / \Gamma)$ are of the form $(\alpha \circ p) \times(\beta \circ q)$ where $\alpha$ and $\beta$ are the eigenfunctions on the manifolds $(M, g)$ and $(N, h)$ respectively. which are invariant under the action by the group $\Gamma$ or takes opposite sign under the action of $\Gamma$.

Proof. Let $\left(U_{i}, \varphi_{i}\right)_{i \in I}$ and $\left(V_{j}, \psi_{j}\right)_{j \in J}$ be two atlas on $(M, g)$ and $(N, h)$ respectively. Then $\left(U_{i} \times U_{j}, \varphi_{i} \times \psi_{j}\right)_{(i, j) \in I X J}$ is an atlas on the Riemannian manifold $(M \times N, g \times h)$. Let $\left(x_{1}, \ldots, x_{n}\right)$ and $\left(y_{1}, \ldots, y_{m}\right)$ be two local coordinate systems on the charts $\left(U_{i}, \varphi_{i}\right)$ and $\left(V_{j}, \psi_{j}\right)$ respectively, where $\operatorname{dim} M=n$ and $\operatorname{dim} N=m$. Then $\left(x_{1}, \ldots, x_{n}, y_{1}, \ldots, y_{m}\right)$ is a local coordinate system on the chart $\left(U_{i} \times V_{j}, \varphi_{i} \times \psi_{j}\right)$, If $\gamma$ is an element of the group $\Gamma$, then we have

$$
\begin{aligned}
& \gamma: M \times N \rightarrow M \times N, \quad \gamma: U_{i} \times V_{j} \rightarrow U_{i}^{\prime} \times V_{j}^{\prime} \\
& \gamma:\left(x_{1}, \ldots, x_{n} ; y_{1}, \ldots, y_{m}\right) \rightarrow\left(\gamma\left(x_{1}, \ldots, x_{n}\right) ; \gamma\left(y_{1}, \ldots, y_{m}\right)\right) \\
& =\left(x_{1}^{\prime}, \ldots, x_{n}^{\prime} ; y_{1}^{\prime}, \ldots, y_{m}^{\prime}\right)
\end{aligned}
$$


which means that $\gamma$ preserves the separate coordinate systems. We assume that

$$
\alpha, \alpha_{1} \in Q_{\lambda}(M, q) \text { and } \beta, \beta_{1}^{\prime} \in Q_{\mu}(N, h)
$$

then we have

$$
\begin{aligned}
& \triangle^{M \times N}[(\alpha o p) \times(\beta o q)]=(\lambda+\mu)[(\alpha o p) \times(\beta o q)] \\
& \triangle^{M \times N}\left[\left(\alpha_{1} o p\right) \times\left(\beta_{1} o q\right)\right]=(\lambda+\mu)\left[\left(\alpha_{1} o p\right) \times\left(\beta_{1} o q\right)\right]
\end{aligned}
$$

If the functions $\alpha$ and $\beta$ are invariant under the action of the group $\Gamma$ and $\alpha_{1}$ and $\beta_{1}$ take opposite sing by the action of the group $\Gamma$, then we have

$$
\begin{aligned}
& \gamma\left(\alpha\left(x_{1}, \ldots, x_{n}\right)\right)=\alpha\left(\gamma\left(x_{1}, \ldots, x_{n}\right)\right)=\alpha\left(x_{1}^{\prime}, \ldots, x_{n}^{\prime}\right)=\alpha\left(x_{1}, \ldots, x_{n}\right) \\
& \gamma\left(\alpha_{1}\left(x_{1}, \ldots, x_{n}\right)\right)=\alpha_{1}\left(\gamma\left(x_{1}, \ldots, x_{n}\right)\right)=\alpha_{1}\left(x_{1}^{\prime}, \ldots, x_{n}^{\prime}\right)=-\alpha_{1}\left(x_{1}, \ldots, x_{n}\right) \\
& \gamma\left(\beta\left(y_{1}, \ldots, y_{m}\right)\right)=\beta\left(\gamma\left(y_{1}, \ldots, y_{m}\right)\right)=\beta\left(y_{1}^{\prime}, \ldots, y_{m}^{\prime}\right)=\beta\left(y_{1}, \ldots, y_{m}\right) \\
& \gamma\left(\beta_{1}\left(y_{1}, \ldots, y_{m}\right)\right)=\beta_{1}\left(\gamma\left(y_{1}, \ldots, y_{m}\right)\right)=\beta\left(y_{1}^{\prime}, \ldots, y_{m}^{\prime}\right)=-\beta_{1}\left(y_{1}, \ldots, y_{m}\right)
\end{aligned}
$$

then we have

$$
\begin{aligned}
& \triangle^{M \times N / \Gamma}[(\alpha o p) \times(\beta o q)]=\triangle^{M \times N}[(\gamma(\alpha) o p) \times(\gamma(\beta) o q)] \\
& =\triangle^{M \times N}[(\alpha o p) \times(\beta o q)]=(\lambda+\mu)[(\alpha o p) \times(\beta o q)] \\
& \triangle^{M \times N / \Gamma}\left[\left(\alpha_{1} o p\right) \times\left(\beta_{1} o q\right)\right]=\triangle^{M \times N}\left[\left(\gamma\left(\alpha_{1}\right) o p\right) \times\left(\gamma\left(\beta_{1}\right) o q\right)\right] \\
& =\triangle^{M \times N}\left[\mid\left(-\alpha_{1} o p\right) \times\left(-\beta_{1} o q\right)\right]=(\lambda+\mu)\left[\left(\alpha_{1} o p\right) \times\left(\beta_{1} o q\right)\right]
\end{aligned}
$$

3. It is known that the Dold manifold is defined by

$$
M^{\prime}=S^{n} \times \mathbb{P}^{m}(\mathbf{C}) / \mathbb{Z}_{2}
$$

where the action of the group $Z_{2}$ is given as follows

$$
\left\{\left(x_{1}, \ldots, x_{n}\right),\left(z_{0}, z_{1}, \ldots, z_{m}\right)\right\} \rightarrow\left\{\left(-x_{1}, \ldots,-x_{n}\right),\left(\bar{z}_{0}, \bar{z}_{1}, \ldots, \bar{z}_{m}\right)\right\}
$$

The manifold $M^{\prime}$ can be obtained by identification of the two points defined by (3.1) on the manifold $S^{n} \times \mathrm{P}^{m}(\mathrm{C})$. 
Theorem 3.1. Let $M^{\prime}=S^{n} \times \mathbb{P}^{m}(\mathbf{C}) / \mathbb{Z}_{2}$ be the Dold manifold provided with the metric $g_{0} \times h_{0}$, where $g_{0}$ is the standard metric on $S^{n}$ with constant sectional curvature 1 and $h_{0}$ is the Study-Fubini metric on $\mathbb{P}^{m}(\mathbf{C})$ with constant holomrphic sectional curvature 1 . Then, the Spectrum of this manifold has the form $S p\left(S^{n} \times \mathbb{P}^{m}(\mathbf{C}) / \mathbb{Z}_{2}, g_{0} \times h_{0} / \mathbb{Z}_{2}\right)=\left\{0, \lambda_{2 k-1}+\mu_{2 \rho-1}, \lambda_{2 k}+\mu_{2 \rho-1}, \lambda_{2 k}+\right.$ $\mu_{2 \rho}$, where $\lambda_{\nu}=\nu(n+\nu-1), \mu_{\sigma}=\sigma(m+\sigma)$ with multipilicities $q_{\nu}\left(\lambda_{\nu}\right)=$ $\frac{(n+\nu-2) \ldots(n+1) n(n+2 \nu-1)}{\nu !}, q_{\sigma}\left(\mu_{\sigma}\right)=A(-m, \sigma)$ if $\sigma$ is odd and $q_{\sigma}\left(\mu_{\sigma}\right)=2 A(m, \sigma)$, if $\sigma$ is even, where $A(m, \sigma)=\frac{2(2 m+\sigma-1)(2 m+\sigma) \ldots(2 m+2)(2 m+1)(m+\sigma)}{\sigma !}$, respectively.

Proof. If is known the eigenfuctions of $\triangle^{S^{n}}$ can be obtained by the restriction on $S^{n}$ the harmonic polynomials on $\mathbb{R}^{n+1}$. The harmonic polynomials which are invariant under the action of the group $\mathbb{Z}_{2}$ are the even dimensional. The harmonic polynomials which take opposite sign by the action of the group $\mathbb{Z}_{2}$ are the odd dimension. Finally, we conclude that all the harmonic polynomials of $\mathbb{R}^{n+1}$ give eigenvalues for $\triangle^{S^{n} \times \mathbb{P}^{m}}(C) / \mathbf{Z}_{2}$.

Therefore we obtain

$$
\lambda_{k}=k(n+k-1), q_{k}\left(\lambda_{k}\right):(n+k-2) \cdots(n+1) n(n+2 k-1) / k !
$$

The complex projective space $\mathbf{P}^{m}(\mathbf{C})$ is the base space of the following fibre

$$
S^{1} \stackrel{i}{\longrightarrow} S^{2 m+1} \stackrel{\pi}{\longrightarrow} \mathbb{P}^{m}(\mathrm{C})
$$

Therefore the eigenfunctions of $\triangle^{\mathbb{P}^{m(\mathbf{C})}}$ are the eigenfunctions of $S^{2 m+1}$ which are invariants by the action of $S^{1}$, or the harmoric polynomials in $\mathbb{R}^{2 m+2} \simeq \mathrm{C}^{m+1}$ which are invariant by the action of $S^{1}$. At the same time we try to determine which of these polynomials are invariants, by the action of the conjugation and the others taking opposite sign by conjugation.

If $\left(z_{0}, z_{1}, \ldots, z_{m}\right)$ and $\left(x_{0}, x_{1}, \ldots, x_{m}, y_{0}, y_{1}, \ldots, y_{m}\right)$ are the natural coordinate systems on $\mathbb{C}^{m+1}$ and $\mathbb{R}^{2 m+2}$ respectively, then we have the relations

$$
z_{0}=x_{0}+\sqrt{-1} y_{0}, z_{1}=x_{1}+\sqrt{-1} y_{1}, \ldots, z_{m}=x_{m}+\sqrt{-1} y_{m}
$$


From (3.5) we obtain

$$
\bar{z}_{0}=x_{0}-\sqrt{-1} y_{0}, \bar{z}_{1}=x_{1}-\sqrt{-1} y_{1}, \ldots ' \bar{z}_{m}=x_{m}-\sqrt{-1} y_{m}
$$

The relations (3.5) and (3.6) imply

$$
\begin{aligned}
& x_{0}=\frac{1}{2}\left(z_{0}+\bar{z}_{0}\right), x_{1}=\frac{1}{2}\left(z_{1}+\bar{z}_{1}\right), \ldots, x_{m}=\frac{1}{2}\left(z_{m}+\bar{z}_{m}\right) \\
& y_{0}=\frac{1}{2 \sqrt{-1}}\left(z_{0}-i \bar{z}_{0}\right), y_{1}=\frac{1}{2 \sqrt{-1}}\left(z_{1}-i \bar{z}_{1}\right), \ldots, y_{m}=\frac{1}{2 \sqrt{-1}}\left(z_{m}-i \bar{z}_{m}\right)
\end{aligned}
$$

Therefore $\left(z_{0}, z_{1}, \ldots, z_{m}, \bar{z}_{0}, \bar{z}_{1}, \ldots, \bar{z}_{m}\right)$ can be obtained as a coordinate system on $\mathbb{R}^{2 m+2}$. The harmonic polynomials on $\mathbb{R}^{2 m+2} \simeq \mathrm{C}^{m+1}$ are polynomials in $z=\left(z_{1}, z_{2}, \ldots, z_{m}\right)$ and in $\bar{z}=\left(\bar{z}_{0}, \bar{z}_{1}, \ldots, \bar{z}_{m}\right)$. The Laplace operator on $\mathbb{R}^{2 m+2}=\mathbb{C}^{m+1}$ has the form

$$
\Delta=-\frac{1}{4} \sum_{p=1}^{m+1} \frac{\partial}{\partial z_{p}} \frac{\partial}{\partial \bar{z}_{p}}
$$

The polynomial $Q=Q(z, \bar{z})=Q\left(z_{0}, z_{1}, \ldots, z_{m}, \bar{z}_{0}, \bar{z}_{1}, \ldots, \bar{z}_{m}\right)$ is harmonic if we have

$$
\sum_{p=1}^{m+1} \frac{\partial^{2}}{\partial z_{p}} \frac{Q}{\partial \bar{z}_{p}}=0
$$

The harmonic polynomials $Q(z, \bar{z})$, which are invariant by the action of $S^{1}$ are the same degree with respect to $z$ and $\bar{z}$. All these polynomials are denoted by $H_{p, p}$. Therefore the eigenvalue which corresponds to each of the eigenfunctions of $\Delta^{S^{2 m+1}}$ which are at the same time eigenfunctions of $\Delta^{P^{m}(C)}$ has the form

$$
\mu_{p}=2 p(2 m+1+2 p-1)=4 p(m+p), \quad p \geq 0 .
$$

From these polynomials we must take those remaining invariant by conjugation and the others taking opposite sign by conjugation. The first polynomials form a vector space of the same dimension as the vector space $H_{p}$, when $p$ is odd. The other polynomials, when $p$ is even, form a vector space whose dimension is $2 \operatorname{dim} H_{p}$. Therefore, we have the following eigenvalues for the Dold manifold 
$\triangle^{S^{n}} \times \mathbb{P}^{m}(\mathbf{C}) / \mathbb{Z}_{2}:$

$0, \lambda_{2 k-1}+\mu_{2 p-1}, \lambda_{2 k}+\mu_{2 p-1}, \lambda_{2 k}+\mu_{2 p}$, where $\lambda_{\nu}=\nu(\nu+n-1), \mu_{\sigma}=4 \sigma(\mu+\sigma)$ with multiplicities $q_{k}\left(\lambda_{k}\right)=(n+k-2) \ldots(n+1) n(n+2 k-1) / k !, q_{\sigma}\left(\mu_{\sigma}\right)=$ $2 A(m, \sigma)$ if $\sigma$ is even and $q_{\sigma}\left(\mu_{\sigma}\right)=A(m, \sigma)$ if $\sigma$ is odd, where $A(m, \sigma)=$ $\frac{2(2 m+p-1) \ldots(2 m+1)(m+p)}{p !}$.

4. Let $\left(M \times N, g_{1} \times g_{2}\right)$ be a compact and orientable Riemannian manifold with the following properties

(i) $N$ can carry a complex structure,

(ii) $\pi_{1}(M \times N)=\mathbb{Z}_{2}$

(iii) $S p\left(M \times N, g_{1} \times g_{2}\right)=S p\left(S^{n} \times \mathbb{P}^{m}(\mathbf{C}) / \mathbb{Z}_{2}, g_{0} \times h_{0} / \mathbb{Z}_{2}\right)$

(vi) $g=$ Kähler metric

We give the conditions under which the Riemannian manifolds ( $M \times N, g_{1} \times$ $\left.g_{2}\right)$ and $\left(S^{n} \times \mathbb{P}^{m}(\mathbb{C}) / \mathbb{Z}_{2}, g_{0} \times h_{0} / \mathbb{Z}_{2}\right)$ are isometric.

It is known that the universal covering of the manifold $S^{n} \times \mathbb{P}^{m}(\mathbb{C}) / \mathbb{Z}_{2}$ is $S^{n} \times \mathbb{P}^{m}(\mathbb{C})$. We denote by $M^{\prime} \times N^{\prime}$ the universal covering of $M \times N$.

These manifolds $M^{\prime} \times N^{\prime}$ and $S^{n} \times \mathbb{P}^{m}(\mathbb{C})$ have metrics $g_{1}^{\prime} \times g_{2}^{\prime}$ and $g_{0} \times h_{0}$ respectively such that $g_{1}^{\prime} \times g_{2}^{\prime} / \mathbb{Z}_{2}=g_{1} \times g_{2}$. The manifolds $\left(M \times N, g_{1} \times g_{2}\right)$ and $\left(S^{n} \times \mathbb{P}^{M}(\mathbb{C}) / \mathbb{Z}_{2}, g_{0} \times h_{0} / \mathbb{Z}_{2}\right)$ are locally isometric onto the manifolds $\left(M^{\prime} \times\right.$ $\left.N^{\prime}, g_{1}^{\prime} \times g_{2}^{\prime}\right)$ and $\left(S^{n} \times \mathbb{P}^{m}(\mathbb{C}), g_{0} \times h_{0}\right)$ respectively. It can easily obtained from $S p\left(M \times N, g_{1} \times g_{2}\right)$ and $S p\left(S^{n} \times \mathbb{P}^{m}(\mathbf{C}) / \mathbb{Z}_{2}, g_{0} \times h_{0} / \mathbb{Z}_{2}\right)$ that $S p\left(S^{n} \times\right.$ $\left.\mathbb{P}^{m}(\mathrm{C}), g_{0} \times h_{0}\right)$ and $S p\left(M^{\prime} \times N^{\prime}, g_{1}^{\prime} \times g_{2}^{\prime}\right)$ have the same eigeuvalues. We assume that these eigenvalues have the same multiplicity and therefore we obtain

$$
S p\left(M^{\prime} \times N^{\prime}, g_{1}^{\prime} \times g_{2}^{\prime}\right)=S p\left(S^{n} \times \mathbb{P}^{m}(\mathrm{C}), g_{0} \times h_{0}\right)
$$

From (4.2) we obtain

$$
\begin{aligned}
& S p\left(M^{\prime}, g_{1}^{\prime}\right)=S p\left(S^{n}, g_{0}\right) \\
& S p\left(N^{\prime}, g_{2}^{\prime}\right)=S p\left(\mathbb{P}^{m}(\mathbb{C}), h_{0}\right)
\end{aligned}
$$

The relations (4.3) and (4.4) imply $\left(M^{\prime}, g_{1}^{\prime}\right)$ is isometric onto $\left(S^{n}, g_{0}\right)$, if $1 \leq n \leq 6([2])$ and $\left(N^{\prime}, g_{2}^{\prime}\right)$ is beholomorphically isometric onto $\left(\mathbb{P}^{m}(\mathbb{C}), h_{0}\right)$, if $1 \leq m \leq 5,([4])$ 
Now we can state the following theorem .

Theorem 4.1. Let $\left(M \times N, g_{1} \times g_{2}\right)$ be a compact, and orientable Riemannian manifold with the properties $\left((i)-(i v)(4.1)\right.$. Let $\left(M^{\prime} \times N^{\prime}, g_{1}^{\prime} \times g_{2}^{\prime}\right)$ be the universal covering of $M \times N$ such that $g_{1} \times g_{2}=g_{1}^{\prime} \times g_{2}^{\prime} / \mathbb{Z}_{2}$. If $S p(M \times$ $\left.N, g_{1} \times g_{2}\right)=S p\left(S^{n} \times \mathbb{P}^{m}(\mathbf{C}) / Z_{2}, g_{0} \times h_{0} / \mathbb{Z}_{2}\right)$ and the mulitiplicity of the eigenvalues of $S p\left(M^{\prime} \times N^{\prime}, g_{1} \times g_{2}\right)$ are the same as of $S p\left(S^{n} \times \mathrm{P}^{m}(\mathrm{C}), g_{0} \times h_{0}\right)$ and $1 \leq n \leq 6,1 \leq m \leq 5$, Then $\left(M \times N, g_{1} \times g_{2}\right)$ is isometric onto $\left(S^{n} \times \mathbb{R}^{m}(\mathbb{C}) / \mathbb{Z}_{2}, g_{0} \times h_{0} / \mathbb{Z}_{2}\right)$.

If the conditions (4.1) are substituded by the following

(i) $N$ is beholomorphically isomorphic onto $\mathbb{P}^{m}(\mathrm{C})$

(ii) $S p\left(M \times N, g_{1} \times g_{2}\right)=S p\left(S^{n} \times \mathbb{P}^{m}(\mathbf{C}), g_{0} \times h_{0} / \mathbb{Z}_{2}\right)$

(iii) Multiplity of the eigenvalues of $S p\left(M^{\prime} \times N^{\prime}, g_{1} \times g_{2}\right)$ are the same as of $S p\left(S^{n} \times \mathbb{P}^{m}(\mathrm{C}), g_{0} \times g_{0}\right)$ then $\left(N^{\prime}, g^{\prime}\right)$ coincides with $\left(\mathbb{P}^{m}(\mathrm{C}), h_{0}\right)$.

From the theorem (4.1) and the conditions (4.5) we conclude the following corollary.

Corollary 4.2. Let $\left(M \times N, g_{1} \times g_{2}\right)$ be a compact and orientable Riemannian manifold with the propporties (4.5), If $S p\left(M \times N, g_{1} \times g_{2}\right)=S p\left(S^{n} \times\right.$ $\left.\mathbb{P}^{m}(\mathrm{C}) / \mathbb{Z}_{2}, g_{0} \times h_{0} / \mathbb{Z}_{2}\right)$, where $2 \leq n \leq 6$, and $1 \leq m \leq 5$, then $\left(M \times N, g_{1} \times g_{2}\right)$ is isometric onto $\left(S^{n} \times \mathbb{P}^{m}(\mathbf{C}), g_{0} \times h_{0}\right)$.

$\S 5$. The manifold $S^{2 n-1} \times \mathrm{P}^{m}(\mathrm{C}) / \Gamma$ is called generalized Dold manifold, where Gamma is a finite subgroup of order greater or equal than three of the group of isommetries $I\left(S^{2 n-1} \times \mathrm{P}^{m}(\mathrm{C})\right)$ of the manifold $S^{2 n-1} \times \mathrm{P}^{m}(\mathrm{C})$.

If we use the same technique as for the manifold $S^{n} \times \mathrm{P}^{m}(\mathrm{C}) / Z_{2}$ then we can compute the $S_{p}\left(S^{2 n-1} \times \mathrm{P}^{m}(\mathbf{C}) / \Gamma\right)$.

Now we can prove the theorem:

Theorem 5.1. Let $S^{2 n-1} \times \mathrm{P}^{m}(\mathrm{C}) / \Gamma$ be a generalized Dold manifold. If $n, m$ and order of $\Gamma$ have special prices, then $S_{p}\left(S^{2 n-1} \times \mathbb{P}^{m}(\mathrm{C}) / \Gamma\right)$ delermines the geometry on the manifold $S^{2 n-1} \times \mathrm{P}^{m}(\mathrm{C})$ ). 


\section{References}

[1] M. Apte, "Sur certaines classes caractéristiques des variétes Kählériennes compactes", $C$. R. A. S., 240, p. 149, 1955.

[2] M. Berger, P. Gandachon and E. Mozet, "Le spectre d' une variéte Riemannienne", Lecture Notes in Mathematics Vol. 194, Springer-Verlag Berlin and New York, 1971.

[3] F. Hirzebruch, Topological methods in Algebraic Geometry, Springer-Verlag, New York, 1966.

4] S. Tanno, "Eigenvalues of the Laplacian of Riemannian manifolds", Tôhoku Math. Jour. 25, pp. 391-403, 1973.

Themistokleous 21, Trikala 42.100, Greece. 